\title{
Editorial: General specialists
}

\author{
Akhlesh Lakhtakia \\ Editor-in-Chief
}

Even before moving to Pennsylvania almost three decades ago, I had admired Benjamin Franklin. Not only was he an author, publisher, diplomat, postmaster, political theorist, and one of the founding fathers of USA, but he was also a first-rate technoscientific researcher. Anyone who has seen the numerous fierce storms that sweep the Atlantic seaboard would be glad to learn that Franklin invented the lightning rod. He also invented bifocal glasses, a stove that heats better than an open fireplace yet spews out much less smoke, and a flexible urinary catheter for immobile patients - all devices in common use more than two centuries later. He was the first to map the Gulf Stream to produce navigation charts that trimmed the transatlantic sailing time by two weeks. Spreading snatches of cloth of different colors on snow, he determined the scientific reason for wearing dark clothes in winter to keep warm and light clothes in summer to keep cool. With his most famous experiment that gave him a frightening jolt, he ascertained that lightning is electricity.

Franklin was not alone among his technoscientific contemporaries for the diversity of his research activities. Perhaps the most prolific mathematician ever, Leonhard Euler originated graph theory which underlies transmission networks, and also developed the equations of inviscid flow. Siméon Poisson was not only an astronomer and a geometer, but he also conjured up the basic differential equation of potential theory and a distribution of probabilities, along with developing the rational use of momentum in mechanical analysis. And how can the great Carl-Friedrich Gauss be forgotten for his seminal contributions to practically every branch of mathematics and physical sciences?

That which today is called multidisciplinarity remained in vogue until perhaps the 1910s. My own hero, James Clerk Maxwell, fathered the fundamental set of electromagnetic equations, a probability density distribution in thermodynamics, color photographs, and even a centrifugal governor for steam engines. Jagadis Chunder Bose carried out pioneering investigations on microwave materials at frequencies as high as $60 \mathrm{GHz}$; invented artificial chiral composite materials; designed, fabricated, and tested structurally chiral materials; devised semiconductor heterojunctions to detect radio signals; and invented the crescograph to identify the similarities of plant and animal tissue.

The rise of the specialist probably occurred in the early 20th century, quite possibly because of the spectacular growth in scientific activity worldwide. Ever more departments and academic majors, each associated with a narrow discipline, were created at universities. Perhaps this trend was led by the Nordic and central European countries, as the names of university departments there would confirm. Perhaps universities in USA were the bellwethers instead. International agencies quickly caught up with the trend, as did also newly independent nations and communist countries. To be sure, there were also those who despised compartmentalization, the medical humorist Richard Gordon among them: "None of [the Yanks] would think of blowing their noses or changing the baby's nappies without first consulting the appropriate specialist-all American doctors of course being specialists. I tell them I'm a general specialist, and that seems to do the trick."

Necessity being a parent of innovation, narrow compartmentalization is at odds with the broad requirements of need-driven technoscientific research. No wonder, as early as during the 1980s, universities began supplementing the hierarchy of colleges and departments with research centers that cut across disciplinary boundaries. Nowadays, national and international

1934-2608/2011/\$25.00 @ 2011 SPIE 
scientific foundations often explicitly require the formation of multidisciplinary research teams, sometimes across different universities and even countries.

No complex engineered system can be the exclusive domain of a certain specialization. The attentions of mechanical engineers, chemical engineers, materials scientists, electrical engineers, civil engineers, fluid mechanicians, and ergonomists, not to mention artists, stylists, and market researchers, are required today to manufacture as commonplace a device as an automobile. The same multidisciplinarity is in evidence during the construction of modern single-family dwellings and multi-story apartment buildings.

At the technoscientific forefront, multidisciplinarity is nowhere more evident than in nanotechnology. Whenever just a few molecules or multimolecular clusters have to be manipulated, economies of activity and functionality rule. Manipulation of extremely small amounts of matter over very tiny distances requires many different types of talents, and many different phenomenons have to be managed to extract the display of an unusual set of functionalities by the final product. Crossover of expertise then has to occur.

Nanotechnology is turning us into general specialists! 